\title{
Cómo pensar e investigar cuando el Estado se retira
}

Miguel A. García

Universidad de Buenos Aires

Consejo Nacional de Investigaciones Científicas y Técnicas, Buenos Aires, Argentina

magarcia@conicet.gov.ar

Recepción: septiembre 2020. Aceptación: diciembre 2020.

\section{Resumen}

El artículo discute las consecuencias de las políticas públicas aplicadas por el neoliberalismo en el campo de la investigación en las Ciencias Sociales y las Humanidades y, en particular, en las áreas de las disciplinas que se interesan por las expresiones musicales y sonoras. La discusión se limita a la Argentina y al período 2016-2019, en el cual gobernó una alianza de neoliberales y conservadores que llevó al poder, mediante elecciones ganadas con falsas promesas y fake news ampliamente difundidas por los medios de comunicación afines, a un grupo de empresarios y directores ejecutivos de grandes empresas.

Palabras clave: investigación en música, Argentina, neoliberalismo, políticas públicas, Estado

\section{Como pensar e investigar quando o Estado se retira}

\section{Resumo}

Este artigo discute as consequências das políticas públicas aplicadas pelo neoliberalismo no campo da investigação em Ciências Sociais e Humanidades e, em particular, em áreas disciplinares que se interessam pelas expressões musicais e sonoras. A discussão refere-se ao período entre 2016 e 2019 na Argentina, durante o qual o país foi governado por uma aliança de perfil neoliberal e conservador. Eleita através de uma campanha alimentada por falsas promessas e fake news, amplamente difundidas 
pelos meios de comunicação alinhados, esta aliança conduziu ao poder um grupo de empresários e diretores executivos de grandes empresas e à consequente retirada do Estado.

Palavras chave: investigação em música, Argentina, neoliberalismo, políticas públicas, Estado

\title{
How to Think and Do Research Work When the State Retreats
}

\begin{abstract}
The article discusses the consequences of the public policies applied by neoliberalism in the research field of social sciences and the humanities and, in particular, in the areas of those disciplines interested in music and sound expressions. The discussion limits itself to Argentina and to the 2016-2019 period, during which an alliance of neoliberals and conservatives ruled, and which brought to power a group of businesspeople and CEOs from big corporations by means of elections won with false promises and fake news widely spread by allied mass media.
\end{abstract}

Keywords: Music research, Argentina, neoliberalism, public policies, State

Los gobiernos neoliberales que surgieron en los últimos años en varios países de América Latina están llevando a cabo drásticas reducciones presupuestarias en el ámbito de la investigación científica y en la promoción de las artes. En el caso de las Ciencias Sociales y las Humanidades, esas reducciones intentan justificarse mediante descalificaciones pergeñadas con información falsa que buscan convencer a la opinión pública sobre su inutilidad e instalar la idea de que la inversión en el sector es, en el mejor de los casos, un despropósito. El campo que comprende la investigación y la enseñanza de la música no resulta indemne de esas reducciones. Las políticas de Mauricio Macri en Argentina y de Jair Bolsonaro en Brasil evidencian que la desfinanciación de la investigación científica y del fomento de las artes es solamente una parte de un proyecto que acelera obscenamente la concentración de la riqueza, facilita la fuga de capitales y propicia el desentendimiento del Estado en cuestiones críticas referidas a la pobreza y al acceso a la salud y educación. En la páginas que siguen ofrezco un breve panorama de las instituciones que financian las investigaciones en música en Argentina y resalto, a la luz de las estrategias desplegadas por 
el neoliberalismo, las consecuencias de las políticas públicas del sector implementadas durante los últimos años.

\section{Las instituciones}

En Argentina existen varias instituciones públicas que fomentan las investigaciones en Ciencias Sociales y Humanidades a través de puestos de trabajo para investigadores/as, financiación de proyectos de investigación (de dos a cinco años de duración), becas doctorales y posdoctorales, y subsidios para realizar viajes al exterior de intercambio académico, entre otros medios. El principal organismo de promoción de la ciencia y la tecnología, en cuanto a su tipo y a sus dimensiones, es el Consejo Nacional de Investigaciones Científicas y Técnicas (CONICET), ente autárquico que se encuentra bajo la órbita del Ministerio de Ciencia, Tecnología e Innovación. El CONICET se creó el 5 de febrero de 1958 y su primer director fue el médico y farmacéutico Bernardo A. Houssay (1887-1971), quien en 1947 obtuvo el Premio Nobel de Medicina.

En la actualidad, el CONICET alberga alrededor de diez mil investigadores/ as (de planta), doce mil becarios/as de doctorado y posdoctorado, 2.600 técnicos/as y profesionales de apoyo a la investigación y 1.500 empleados/ as administrativos/as. Como se informa en su página Web, ${ }^{1}$ la totalidad del personal de la institución trabaja repartidos en quince Centros Científicos Tecnológicos (CCT), once Centros de Investigaciones y Transferencia (CIT), un Centro de Investigación Multidisciplinario, 280 Institutos y Centros exclusivos del CONICET -de doble y triple dependencia con universidades nacionales y otras instituciones- $y$ en otros Centros que son exclusivos de las universidades. Sus actividades se desarrollan en las llamadas "grandes áreas del conocimiento”, estas son: Ciencias Agrarias, Ingeniería y de Materiales; Ciencias Biológicas y de la Salud; Ciencias Exactas y Naturales; y Ciencias Sociales y Humanidades. Los/as investigadores/as y becarios/ as del área de Ciencias Sociales y Humanidades corresponden a la cuarta parte del total que posee el organismo. Esta área, precisamente, acoge las investigaciones sobre música, tanto aquellas que se desarrollan en el marco de la etnomusicología, la musicología histórica, la sociología de la música, los estudios de música popular como de otras disciplinas con intereses cercanos.

${ }^{1}$ https://www.conicet.gov.ar 
Otro organismo autárquico, que también actúa bajo la esfera del Ministerio de Ciencia, Tecnología e Innovación es la Agencia Nacional de Promoción de la Investigación, el Desarrollo Tecnológico y la Innovación. ${ }^{2}$ La Agencia, creada el 27 de diciembre de 1996 por decreto del Poder Ejecutivo Nacional como Agencia Nacional de Promoción Científica, promueve la investigación científica, la generación de conocimiento y la innovación productiva mediante el otorgamiento de fondos, mayormente provenientes del exterior, a investigadores/as, instituciones y empresas. Los fondos proceden de distintas fuentes: del Estado Nacional, del Banco Interamericano de Desarrollo, del Banco Internacional de Reconstrucción y Fomento, del Banco de Desarrollo de América Latina, del Banco Centroamericano de Integración Económica y también de reembolsos de distintos convenios de cooperación que la Agencia mantiene con instituciones y empresas del país y del exterior.

Otras instituciones que financian investigaciones sobre música son las universidades nacionales. En realidad, solo en algunas de las 56 Universidades Nacionales ${ }^{3}$ que tiene el país, se llevan a cabo ese tipo de investigaciones. En ellas tienen sede de trabajo gran parte de los/as investigadores/ as del CONICET, además de docentes-investigadores/as que pertenecen exclusivamente a esas casas de estudio. Las universidades promueven las investigaciones principalmente mediante el otorgamiento de becas, financiación de proyectos y entrega de subsidios para viajes, organización de congresos, publicaciones, compra de equipamiento y cargos docentes con dedicación parcial a la investigación.

Entre las instituciones que se dedican exclusivamente a la investigación sobre música, la de mayor importancia es el Instituto Nacional de Musicología "Carlos Vega" (INM), ${ }^{4}$ organismo dependiente del Ministerio de Cultura de la Nación. El INM se creó a partir de varias instituciones, la primera de ellas, acaecida en 1931 por iniciativa del musicólogo Carlos Vega, fue el Gabinete de Musicología Indígena con sede en el Museo de Ciencias Naturales "Bernardino Rivadavia”. Con posterioridad, el Gabinete adquirió los nombres de Instituto de Musicología (1944), Instituto Nacional de Musicología (1971) e Instituto Nacional de Musicología "Carlos Vega" (1973). Se trata de una institución dedicada a la investigación musicológica en sentido amplio, aunque por muchos años sus focos de interés fueron las músicas y danzas criollas de raigambre rural y las músicas de

\footnotetext{
${ }^{2}$ http://www.agencia.mincyt.gob.ar/index.php

${ }^{3}$ Ver lista de universidades en https://www.cin.edu.ar/instituciones-universitarias/

${ }^{4}$ https://inmcv.cultura.gob.ar
} 
los pueblos originarios, de lo cual da cuenta la abundancia y riqueza de los fondos documentales que posee, originados en trabajos de campo llevados a cabo en Argentina y países limítrofes.

Asimismo, deben mencionarse otras dos instituciones dedicadas a la investigación. Una de ellas es Instituto de Investigación en Etnomusicología, ${ }^{5}$ creado en 2006 en la esfera administrativa del Ministerio de Cultura de la Ciudad de Buenos Aires, que tiene en custodia diversos fondos documentales y propicia investigaciones tanto en el ámbito de la etnomusicología como en los de los estudios de músicas populares y académicas. La otra institución pertenece al ámbito privado. Se trata del Instituto de Investigación Musicológica Carlos Vega, que tiene sede en la Facultad de Artes y Ciencias Musicales de la Pontificia Universidad Católica Argentina y que fue creado en 1966 a partir de la donación de Carlos Vega de manuscritos inéditos, sus epistolario y archivo personal. ${ }^{6}$ Además de las instituciones mencionadas hay que nombrar a otras dos que, aunque en menor medida, también fomentan investigaciones en el área mediante subsidios; estas son el Fondo Nacional de las Artes ${ }^{7}$ y el Fondo Metropolitano de la Cultura, las Artes y las Ciencias del Gobierno de la Ciudad de Buenos Aires. ${ }^{8}$ En esta lista tampoco puede omitirse a la Asociación Argentina de Musicología (AAM), ${ }^{9}$ asociación civil creada en 1985 , que, a pesar de no financiar en forma directa proyectos de investigación, tiene un rol destacado en la promoción del área a través de sus conferencias, publicaciones y página Web.

\section{Políticas de inversión y desinversión}

La asignación de fondos para todas estas instituciones y sus consecuentes expansiones y retracciones estuvieron sujetas a los posicionamientos ideológicos de las fuerzas políticas que gobernaron el país. En el escenario de los últimos quince años se aprecia cómo bajo la égida de un gobierno progresista, en el período 2003-2015, el área de ciencia y tecnología fue promocionada con fuertes inversiones de capital, aumento de recursos humanos y jerarquización de sus instituciones; mientras que bajo la

\footnotetext{
5 https://www.buenosaires.gob.ar/cultura/ensenanza-artistica/instituto-de-investigacion-en-etnomusicologia

${ }^{6}$ http://uca.edu.ar/es/facultad-de-artes-y-ciencias-musicales/instituto-de-investigacion-musicologica-carlos-vega

${ }^{7}$ https://fnartes.gob.ar

${ }^{8}$ https://www.buenosaires.gob.ar/fondometropolitano

${ }^{9}$ https://www.aamusicologia.org.ar
} 
administración neoliberal, en el período 2016-2019, la política del sector estuvo dirigida a su vaciamiento y desprestigio.

Observemos con un poco más de detalle estas diferencias. En el período 2003-2015, el estado argentino ascendió a rango de Ministerio la Secretaría de Ciencia y Técnica, dando lugar a la creación, en el año 2007, del Ministerio de Ciencia, Tecnología e Innovación Productiva (MINCyT). Esta institución fue el principal actor de una política que benefició el sistema científico-tecnológico mediante el aumento de ingresantes a la carrera de investigador/a del CONICET, de líneas de financiamiento para la investigación, del número de becas de grado y posgrado, y la cantidad de personal técnico y administrativo. Asimismo puso en marcha medidas que favorecieron la repatriación de científicos/as e hizo una inversión cuantiosa en la construcción de 90.000 metros cuadrados de laboratorios y del Polo Científico Tecnológico; edificio que es la sede administrativa de varias instituciones relacionadas con la ciencia y la tecnología. ${ }^{10}$ Esta expansión del sistema científico fue acompañada de una política redistributiva que benefició a los sectores más desfavorecidos mediante el fortalecimiento de la salud y la educación pública, y de un conjunto de medidas dirigidas a facilitar el acceso al mercado laboral. Paralelamente se produjo una extraordinaria ampliación de derechos (como la sanción de la Ley de matrimonio igualitario) y un fuerte proceso de politización de la población que puso en agenda discusiones sobre la perspectiva de género, la interrupción voluntaria de embarazo, los derechos de las minorías, la discriminación, la ética de la comunicación e incluso sobre el rol que debían cumplir la ciencia y la tecnología en el desarrollo del país, entre otras cuestiones.

En el período 2016-2019, gobernado por una alianza de neoliberales y conservadores que llevó al poder, mediante elecciones ganadas con falsas promesas y fake news, a un grupo de empresarios/as y directores/as ejecutivos/as de grandes empresas, tuvo lugar, como fue dicho, una acelerada retracción de todos los recursos del sistema científico y tecnológico. La medida más representativa y de mayor violencia simbólica de la política del gobierno de Mauricio Macri fue la degradación del Ministerio de Ciencia, Tecnología e Innovación Productiva (MINCyT) al rango de Secretaría. A esta medida le siguió una fuerte desinversión del sistema científico que, en el caso del CONICET, significó una pérdida del poder adquisitivo de los salarios de aproximadamente 30\% (en los cuatro años del gobierno de Macri), reducción del presupuesto para los institutos (algunos llegaron

${ }^{10}$ Para mayor información sobre estos aspecto puede consultarse Alasino (2020). 
a tener problemas para afrontar el pago de los servicios básicos de electricidad, gas, etc.), caída del presupuesto para erogaciones en funcionamiento, infraestructura y ejecución de proyectos, reducción del número de vacantes para el ingreso a la Carrera de Investigador, disminución de la cantidad de becas, cancelación de proyectos de cooperación internacional, mora en el pago de las cuotas de los proyectos, etcétera. Un dato más que representativo del desprecio hacia la investigación por parte del modelo neoliberal que gobernó Argentina entre 2016 y 2019 es la magnitud de la pérdida de poder adquisitivo de los montos para investigación que otorga la Agencia Nacional de Promoción Científica: los montos a los que se tenía acceso en el año 2018 fueron ocho veces menores en dólares que aquellos que estaban disponibles en $2010 .^{11}$

Estas políticas de desinversión aplicadas al sistema científico estuvieron acompañadas de falsos discursos que intentaron enfrentar a las ciencias básicas con las ciencias aplicadas y a las "ciencias duras" con las "ciencias blandas", establecer alianzas entre la investigación y el mercado, y mancillar a las Ciencias Sociales y Humanidades por ser éstas el ámbito desde en el cual se gestaban las críticas más incisivas al modelo neoliberal imperante. La pauperización del sistema científico no fue un hecho aislado sino que tuvo su correlato en otros ámbitos de vida del país: destrucción de la educación y la salud pública, aumento de la pobreza, reprimarización de la economía, precarización laboral, endeudamiento externo a razón de más de 30.000 millones de dólares anuales, fuga de capitales, destrucción de las pequeñas y medianas empresas, concentración de la riqueza en bancos, compañías energéticas y empresas cercanas a los funcionarios de turno, represión de la protesta social y uso del lawfare y el espionaje ilegal para extorsionar, acusar y encarcelar a opositores. Es decir, el estrangulamiento del sistema científico corrió en paralelo a la concentración de la riqueza y, por ende, a la exclusión de vastos sectores de la población.

Frente a este escenario, un sector numéricamente significativo de científicos/as, becarios/as, docentes y estudiantes, dispuestos detener o minimizar los estragos que día a día ocasionaba el modelo neoliberal, desplegaron diversas estrategias de organización y movilización. Entre ellas se encuentran el fortalecimiento y la conformación de colectivos científicos, como el grupo Ciencia y Técnica Argentina, ${ }^{12}$ la Red Federal de Afectados (parte de un grupo de 530 ingresantes a la Carrera de Investigador del Conicet que aun con dictamen favorable no fueron incorporados en el 2016), Becarios

\footnotetext{
${ }^{11}$ Mayor información en Aliaga (2019).

${ }^{12}$ https://cienciaytecnicaargentina.wordpress.com
} 
Empoderados, Jóvenes Científicos Precarizados, varios grupos de "autoconvocados" y un movimiento asociativo de directores/as de Institutos del Conicet, entre otros. Estos colectivos propiciaron la realización de encuentros nacionales y regionales, manifestaciones y clases públicas, difusión de solicitadas, amplio uso de las redes sociales para denunciar los recortes presupuestarios, presencia de científicos en los medios opositores al gobierno e incluso dos ocupaciones pacíficas, de una semana de duración cada una, de una parte del edificio del Ministerio de Ciencia, Tecnología e Innovación Productiva. Todas estas acciones llevaron al fortalecimiento de las organizaciones sindicales opositoras con incumbencia en los ámbitos científico, docente y estudiantil, y contaron con gran apoyo de la opinión pública, a pesar de una serie de maniobras del macrismo destinadas desacreditar la labor científica, en particular las áreas de las Ciencias Sociales y Humanidades. El empoderamiento y el prestigio que el sector científico había adquirido durante el período 2003-2015 se mantuvo intacto durante la "ceocracia" macrista y fue un vector de peso dentro las fuerzas que resistieron la pauperización del sector y que condujeron a la derrota del neoliberalismo en las elecciones de octubre de 2019.

\section{Posiciones frente a las políticas públicas del neoliberalismo}

Durante el período 2016-2019, tanto la labor de los/as investigadores/ as como el desempeño de las instituciones dedicadas a la investigación musical marcharon al ritmo de los acontecimientos someramente descriptos en los párrafos anteriores, es decir, debieron sufrir las consecuencias de los recortes presupuestarios: dificultad para participar en congresos internacionales y organizar eventos locales, demora y cancelación de publicaciones, restricciones en la adquisición de equipamiento e insumos y cancelación de contratos laborales. Asimismo, el macrismo intentó imponer en varias dependencias una doctrina mercantil, que incluía el culto a la meritocracia, el emprendedurismo y el coaching, a fin de reorientar sus actividades hacia el campo de la comunicación y el marketing. La construcción de una imagen institucional basada en la eficiencia y la modernización intentó desplazar las actividades de investigación en sí mismas. Pero ese plan, que quiso aplicarse al Instituto Nacional de Musicología "Carlos Vega”, entre otras instituciones, alcanzó poco más que su enunciación porque se dio de bruces con la paupérrima inversión que el gobierno destinaba al sector. No obstante, tuvo el efecto deseado de operar como una cortina de humo que, aunque tenue y efímera, logró obnubilar a algunos/as investigadores/as impidiéndoles vislumbrar que tras la 
humareda se horadaba el campo de la investigación y en el plano de la macroeconomía se transferían miles de millones de dólares al exterior y se iba rápida y directamente al default. Sin embargo, las consignas vacuas de la "revolución de la alegría", la modernización y de otros espejismos igualmente falaces que ornamentaban el discurso macrista, no lograron engañar a la totalidad de la "comunidad musicológica". Durante el período en cuestión la Asociación Argentina de Musicología, institución que reúne una porción considerable de los investigadores/as del país que trabajan en las diversas vertientes de los estudios sobre música, estuvo gestionada por tres Comisiones Directivas, una en el bienio 2015-2016, otra en 2017-2018 y la última en 2019-2020. Estas Comisiones tuvieron posiciones disímiles frente al nuevo escenario diseñado por las políticas neoliberales. La primera Comisión se mostró renuente a manifestar su repudio a los despidos y recortes presupuestarios, y aun a convocar una reunión de socios/as a los fines de discutir el problema -en realidad no todos sus integrantes comulgaban con esa postura. Esto produjo un agudo cruce de opiniones en la lista de discusión entre simpatizantes del gobierno que celebraban todas sus medidas a la par de proclamar la falsa disociación entre academia y política o de confundir reclamos del sector con partidismo, y opositores/ as que se resistían a la retracción del área y vislumbraban la hecatombe que se avecinaba. Un ambiente enrarecido ocasionado por los despidos $\mathrm{y}$ otros actos de violencia institucional fueron las principales causas de que un grupo importante de los/as socios/as, apático o temeroso de ser perjudicado por develar su afinidad con una u otra posición, optara por callar o por expresar sus opiniones en forma privada. Las Comisiones que dirigieron la AAM durante los bienios 2017-2018 y 2019-2020 mantuvieron una posición diferente: llevaron adelante una explícita defensa del área y se mostraron solidarias con lo que sucedía en otros campos y dependencias que estaban siendo afectadas por las mismas políticas.

Estos acontecimientos pasaron desapercibidos para gran parte de quienes se desenvolvían en las Ciencias Sociales y Humanidades debido en buena medida al desarrollo acotado que tienen los estudios de música en la Argentina. Lamentablemente no hay datos fidedignos de cuántas personas están actualmente trabajando en el área en todo el país. Un conteo manual, al momento de escribir este artículo, de la presencia de investigadores/as y becarios/as del área en el CONICET, que si bien es la institución que más invierte en el área no es la única, arroja un total aproximado de catorce investigadores/as -de carrera y no en su totalidad dedicados/as exclusivamente al campo de la música- y 33 becarios/as. El futuro del área es francamente incierto. No obstante, el gobierno que asumió el poder el 10 de diciembre de 2019, una alianza progresista integrada por fuerzas 
que representan un amplio espectro de centro e izquierda, dio señales prometedoras hacia la recuperación del sistema científico y tecnológico en general, lo cual podría redundar en una mejora para las investigaciones en música. Entre ellas cabe señalar cuatro que son la antítesis de la violencia que ejerció el macrismo hacia la ciencia y la tecnología: la restitución del Ministerio de Ciencia, Tecnología e Innovación Productiva; la reasignación de fondos que estaban destinados a los "gastos reservados" de la Agencia Federal de Inteligencia a ese Ministerio -para desarrollos relacionados con el COVID-19-; la presencia de investigadores/as de las Ciencias Sociales en la primera línea de asesores/as presidenciales y la puesta en marcha de un programa de inversión en infraestructura universitaria que afectará a las 47 universidades nacionales. En el ámbito del CONICET también hubo un cambio de rumbo en la política de inversiones, lo cual se materializó en el aumento en los cupos de ingreso a la Carrera de Investigador/a, en la disponibilidad de becas doctorales y posdoctorales, en las asignaciones monetarias para los proyectos de investigación y en las remuneraciones de las becas doctorales y posdoctorales (el organismo gestiona aproximadamente para todas las áreas 12.000 becas que recibirán en promedio $50 \%$ de aumento).

\section{El credo neoliberal y la conversión de sus víctimas}

Colegas de otras latitudes reconocerán en esta descripción una experiencia cercana, un espejo de lo que sucede en sus países, otros/as, en cambio, advertirán un escenario extraño y perturbador. Estos/as últimos/as tal vez se pregunten con asombro cómo pueden llevarse a cabo investigaciones de manera regular frente a altibajos abruptos y escasa o nula posibilidad de programar el futuro inmediato. No es fácil dar una respuesta concluyente a este interrogante. Lo cierto es que, aun cuando el Estado se retira, seguimos haciendo investigación. Sin duda la falta de financiamiento y el descrédito del área que pregonan las políticas neoliberales producen una retracción en el rendimiento académico. En los tiempos que corren no hay lugar para sostener una perspectiva ascética que considere la austeridad y las crisis como los fundamentos de un terreno fértil para el pensamiento, pues éste hoy requiere múltiples formas de conectividad, movilidad y una infraestructura adecuada y renovable, es decir, demanda inversión. En varios países de Latinoamérica, esa inversión no siempre está garantizada y debe ser suplida, al menos parcialmente, por el uso de versiones gratis e ilegales de software, la descarga ilegal de datos, el máximo aprovechamiento de los sitios con open access y por muchas otras maniobras creativas y de intercambio solidario. 
No deja de inquietar el hecho de que las fuerzas que socavan la investigación en Ciencias Sociales y Humanidades, las mismas que intentan derribar todo aquello que impida o cuestione una rápida y obscena concentración de la riqueza, en las últimas décadas llegan al poder por medio de las urnas. La explicación es sencilla: la voracidad inscripta en los genes del capital logra convertir a su credo, mediante promesas de bienestar, justicia y transparencia que sus artífices ni remotamente pensaron en cumplir, a sectores subalternizados y no tan subalternizados que tras la farsa de un procedimiento electoral devienen sin demora en sus víctimas. Un acontecimiento que patentiza esta situación, y que es relevante para las investigaciones sobre música, es lo que sucedió en el concierto que ofreció Roger Waters en el Allianz Parque de la ciudad de San Pablo, Brasil, en octubre de 2018, en el marco de la gira Us+Them. Como reportaron varios medios internacionales, durante el concierto, Waters incluyó a Jair Bolsonaro, en ese entonces candidato a presidente de Brasil, en una lista de líderes neofascistas. Inesperadamente, una porción significativa del público repudió la actitud de Waters mediante una lluvia de abucheos. El hecho puede comprenderse en el marco de una feroz campaña orquestada por fuerzas neoliberales, grupos de derecha y ultraderecha, líderes religiosos/as y militares que con fake news, procesos judiciales inventados y diversos tipos de artilugios para desprestigiar al Partido dos Trabalhadores, logró convencer a una parte importante del electorado que un giro xenófobo, racista, homófono, militarista y sexista era el camino seguro hacia un orden mejor. En otro lugar (García, 2019) expresé que el neoliberalismo había consumado casi lo imposible: hacer surgir un sujeto esquizoide, que se regocija con el sonido de su ídolo y a la vez se indigna con el contenido de su pensamiento. Probablemente, quienes discreparon con Waters ayudaron en las urnas a forjar el triunfo de Bolsonaro y tal vez también, tristemente fueron o serán víctimas de la política anticientífica e insensible que está teniendo el gobierno de Brasil en la administración de la pandemia del COVID-19.

Otro acontecimiento también significativo para nuestros intereses fue una declaración del presidente de la Fundação Nacional de Artes de Brasil, Dante Mantovani, quien en diciembre de 2019 asoció el rock con el consumo de drogas el sexo, el aborto y el "satanismo". ${ }^{13}$ Su declaración, adornada con referencias poco claras a Herbert Marcuse, Theodor Adorno, John Lennon, la CIA y el gobierno soviético, dio lugar a un repudio generalizado de intelectuales y artistas. Mantovani se expresó no solo en nombre del Estado sino también en el de la academia ya que, como

${ }^{13}$ Me referí previamente al tema en García (2020). 
consta en su página de Internet, posee formación universitaria de grado y posgrado de la Universidade Estadual de Londrina. ${ }^{14}$ El acontecimiento demuestra que en el marco de los gobiernos neoliberales, en este caso ultraderechista, hay lugar para menoscabar no solo lo que hacemos sino también las músicas que estudiamos.

\section{A modo de conclusión: sobre los lugares de enunciación}

Lo expuesto hasta aquí pone en evidencia que las investigaciones en música tienen que eludir innumerables escollos cuando las políticas públicas hacia el sector son pergeñadas con la lógica del mercado. En América Latina esos escollos se originan no solo en recortes presupuestarios y descalificaciones como las ya mencionadas, sino también por la devaluación de las divisas locales que favorece al capital especulativo y perjudica las actividades que requieren la adquisición de bienes y servicios producidos o comercializados en los mercados dolarizados. Los gobiernos neoliberales, representantes y aliados locales del capital transnacional, ayudan a consolidar lo que Syed Farid Alatas llamó "neoimperialismo o neocolonialismo académico" (2003): escenario controlado por un grupo de países liderados por EEUU, Gran Bretaña y Francia, mediante la generación de una gran masa de productos de investigación en la forma de artículos -publicados en revistas con evaluación y en otros medios-, que tienen alcance global, reconocimiento, respeto, prestigio -tanto donde se originan como donde se extienden- y gran capacidad para influenciar las disciplinas sociales. Como explica Eloísa Martín (2018), en el centro de ese escenario se halla la noción de mérito entendida como un atributo que garantiza el éxito académico, el cual se mide por tener publicaciones internacionales que deben estar en inglés y que tienden a asumir teorías y métodos engendrados en el Norte. Este es un juicio generalizado a lo largo y ancho de la academia, y ha estimulado la creación de proyectos para visibilizar trabajos que resultan significativos para las comunidades locales mediante su traducción al inglés. Entre esos proyectos de publicación se encuentran los sitios web de acceso libre Translingual Discourses in Ethnomusicology, ${ }_{15}$ auspiciado por la Univesität Wien y la Kunst-Univesität Graz, y Ethnomusicology Translation, ${ }^{16}$ desarrollado por la Society for Ethnomusicology.

\footnotetext{
$\overline{{ }^{14} \mathrm{https} / / / \text { dantemantovani.org/wp/ }}$

${ }^{15}$ https://www.tde-journal.org/index.php/tde/

${ }^{16}$ https://scholarworks.iu.edu/journals/index.php/emt/index
} 
La conjunción del neoliberalismo económico con el neocolonialismo académico tiene múltiples consecuencias. Por un lado, como fue dicho, restringe el acceso a los recursos necesarios para la investigación y la enseñanza. Por otro lado, aunque la situación es cambiante y presenta varias y complejas aristas, esas restricciones suelen fortalecer una suerte de distribución internacional del trabajo académico en la cual los países que controlan los recursos se abocan mayormente a la producción de teorías y métodos, y el resto a usar esas teorías y métodos para producir estudios de caso. En este sentido, las instituciones académicas de los países mencionados por Alatas (2003) ofrecen un lugar de enunciación confortable, con reputación y abundantes recursos para que quienes escriben desde allí obtengan una cuota de poder que directamente fortalezca la autoridad e influencia de esas instituciones. Ese empoderamiento surge sin importar las nacionalidades de quienes enuncian desde el Norte ni si sus posiciones son celebratorias o críticas hacia el propio lugar de enunciación. El compositor, crítico y musicólogo uruguayo, Coriún Aharonián (1940-2017) retrató con unas pocas pinceladas cómo se reverencia el "santoral académico" impulsado desde el Norte:

Una cosa es la imprescindible costumbre de leer todo lo posible, y de discutirlo, y otra cosa el estar sustituyendo la lectura completa y la discusión comprometida por una praxis de la mención forzada a miembros de un limitado santoral académico, llámense Derrida o Foucault o Lacan o Baudrillard o Deleuze o Althusser o Castoriadis o Guattari o Badiou o Ricoeur, o bien Bajtin o Bauman o Žižek o Vattimo, que aparecen y desaparecen como por arte de magia, pero que curiosamente son casi siempre lanzados desde París (sin que en esto tengan ellos culpa). El alumno universitario es inducido a mencionar dos o tres de esos nombres ya desde la primera página, sin una razón profunda... (2014, p. 133).

Sin duda una asimetría tan polarizada tiene momentos y espacios de fisura. De no ser así estarían negándose desarrollos teóricos y metodológicos locales que, aun en diálogo con ideas gestadas en los centros académicos más influyentes, presentan atributos de originalidad y autonomía. Parte central de la obra del musicólogo argentino Carlos Vega (1898-1966) puede ser considerada un caso de desarrollo autónomo. Vega, aunque inspirado por ideas europeas en boga -algunos aspectos del pensamiento de Gabriel Tarde y de la Escuela histórico-cultural-, logró formular una interpretación propia sobre el surgimiento, la distribución y clasificación de las expresiones musicales del territorio argentino. Su teoría, aunque dé lugar a cuestionamientos por presentar zonas ambiguas y cierta 
subestimación de la capacidad creativa de los sectores populares, tiene una cuota de originalidad y aún hoy goza de cierta aceptación dentro y fuera de Argentina. No obstante, como esta parece ser una excepción a la regla, la idea de una distribución internacional del trabajo académico ligada a una distribución desigual de la riqueza, y que afecta a las investigaciones sobre las músicas, debe ser considerada, al menos, como una hipótesis a explorar. También merece ser revisado el concepto de mérito y cómo éste se fortalece, debilita o recarga de sentido de acuerdo con los cambios de escenarios ideológicos, políticos y económicos.

En un libro de reciente aparición, editado por CLACSO (Basail Rodríguez, 2019) -institución que ha abogado insistentemente por reducir las diferencias en América Latina-, varios autores/as diagnostican la situación de las Ciencias Sociales y las Humanidades en América Latina en términos de asedio y pérdida de la autonomía. Este ha sido el escenario de la investigación de la Argentina cuando el Estado ha aplicado políticas de desinversión del sector. El desafío consiste en quebrar el asedio y recuperar la autonomía reclamando mayor inversión, mejores condiciones y, sobre todo, manteniendo activas la crítica y la autocrítica, explorando nuevas perspectivas, alimentando el ímpetu transdisciplinario que anima nuestras disciplinas desde hace más de un siglo y descolonizando las rutinas de acción y el pensamiento. Todo esto puede ser insuficiente si se confina al olvido el hecho de que cuando el Estado cayó bajo el control del neoliberalismo la labor de las Ciencias Sociales y las Humanidades fue desfinanciada, difamada y amordazada. 


\section{Bibliografía}

» Aharonián, C. (2014). Desde el sur. Entrevista de Omar Corrado. El oído pensante, 2(2),130-144. Recuperado de http://revistascientificas.filo.uba.ar/ index.php/oidopensante/article/view/7447

»Alasino, C. M. (2020). Conicet: una mirada al pasado reciente. Ciencia e Investigación, 70(1), 12-31. Recuperado de https://aargentinapciencias.org/ wp-content/uploads/2020/05/2-Alasino-cei70-1-3.pdf

»Alatas S. F. (2003). Academy Dependecy and the Global Division of Labor in the Social Sciences. Current Sociology, 51, 1-15.

» Aliaga, J. (2019). Algunas propuestas para el sistema de ciencia y técnica argentino. Manuscrito, 48 pp.

» Basail Rodríguez, A. (2019). Academias Asediadas. Convicciones y conveniencias ante la precarización. Chiapas: CLACSO.

»García, M. A. (2019). La seducción del sonido y el desencanto de la palabra. Editorial de El oído pensante, 7(1), 1-3. Recuperado de http:// revistascientificas.filo.uba.ar/index.php/oidopensante/article/ view/7540/6750

" García, M. A. (2020). Contra el fundamentalismo. Editorial de El oído pensante 8(1), 1-3. Recuperado de http://revistascientificas.filo.uba.ar/ index.php/oidopensante/article/view/7594/6792

» Martín, E. (2018). (Re)produção de desigualdades e (re)produção de conhecimento: a presença latino-americana na publicação acadêmica internacional em Ciências Sociais. En Eloísa Martín y Barbara Goebel (Org.). Desigualdades interdependentes e geopolítica do conhecimento: negociações, fluxos, assimetrias, pp. 56-80. Rio de Janeiro: 7 Letras.

\section{Biografía / Biografia / Biography}

\section{Miguel A. García}

Es Doctor en Antropología por la Universidad de Buenos Aires. Actualmente es Investigador Principal del Consejo Nacional de Investigaciones Científicas y Técnicas (CONICET, Argentina) y docente de la cátedra "Antropología de la música" de la Carrera de Artes, de la Facultad de Filosofía y Letras (Universidad de Buenos Aires). Asimismo, es Editor y Director de El oído pensante. Sus investigaciones abordan las siguientes áreas: música de los pueblos originarios del Chaco y Tierra del Fuego, antropología y etnoestética, epistemología de la investigación etnomusicológica, música y colonialismo, archivos sonoros y teoría del archivo. 